\title{
The Effect of Lesions of the Insular Cortex on Instrumental Conditioning: Evidence for a Role in Incentive Memory
}

\author{
Bernard W. Balleine ${ }^{1}$ and Anthony Dickinson ${ }^{2}$ \\ ${ }^{1}$ Department of Psychology, University of California, Los Angeles, Los Angeles, California 90095, and 2Department of \\ Experimental Psychology, University of Cambridge, Cambridge CB2 3EB, United Kingdom
}

\begin{abstract}
In three experiments, we assessed the effect of lesions aimed at the gustatory region of the insular cortex on instrumental conditioning in rats. In experiment 1 , the lesion had no effect on the acquisition of either lever pressing or chain pulling in fooddeprived rats whether these actions earned food pellets or a maltodextrin solution. The lesion did, however, attenuate the impact of outcome devaluation, induced by sensory-specific satiety, on instrumental performance but only when assessed in an extinction test. This effect was not secondary to an impairment in instrumental learning; in experiment 2 , no evidence was found to suggest that the lesioned rats differed from shams in their ability to encode the specific action-outcome contingencies to which they were exposed during training. In experiment 3 ,
\end{abstract}

however, lesioned rats were found to be insensitive to the impact of an incentive learning treatment conducted when they were undeprived; although, again, this deficit was confined to a test conducted in extinction. These results are consistent with the view that, in instrumental conditioning, the gustatory region of the insular cortex is involved in encoding the taste of food outcomes in memory and, hence, in encoding the incentive value assigned to these outcomes on the basis of prevailing motivational conditions.

Key words: instrumental conditioning; gustatory cortex; insular cortex; devaluation; sensory-specific satiety; incentive learning; contingency; motivation; reward
Although the brain mechanisms of reward have long been the subject of intensive investigation (for review, see Robbins and Everitt, 1996), few attempts have been made to specify how rewardrelated processes make contact with responses instrumental to the delivery of rewarding events. Indeed, to the extent that the structure of instrumental conditioning has been specified in the neurobiological literature, it has typically been based on the classic stimulus-response (S-R)/reinforcement system originally advanced within Thorndike's (1911) "law of effect" (Donahoe et al., 1993). There is, however, considerable evidence against this suggestion coming primarily from studies assessing the impact of post-training changes in the reward value of the instrumental outcome on subsequent performance. In one study (Colwill and Rescorla, 1985), for example, hungry rats were trained to lever press and chain pull with one response earning food pellets and the other sucrose solution. After this training, one outcome was devalued using a sensory-specific satiety treatment; i.e., rats were allowed to consume one of the two outcomes, pellets or sucrose, for $1 \mathrm{hr}$, before a choice extinction test conducted on the levers and chains. Although S-R theories predict that this satiety treatment should not produce any differential effect on performance, Colwill and Rescorla (1985) found that, on test, their rats performed fewer of the response and that, in training, had delivered the outcome on which they were sated before the test than of the other response.

A similar devaluation effect has also been reported in rats trained on two levers that both deliver a nutritive outcome differing only with respect to a single taste feature (Balleine and Dickinson, 1998, their Experiment 1), suggesting that, in hungry rats, specific satiety-induced outcome devaluation affects instrumental performance through a change in the attractiveness of taste features of the food outcome. This finding is important because it suggests that processes involved in the detection and representation of taste may

Received May 17, 2000; revised Sept. 15, 2000; accepted Sept. 21, 2000.

This research was supported by National Institute of Mental Health Grant MH56446 and the European Commission Biomed 2 Program. We thank Claire Garner and Szuzana Varga for their assistance in conducting these experiments.

Correspondence should be addressed to Bernard W. Balleine, Department of Psychology, University of California, Los Angeles, Box 951563, Los Angeles, CA 90095-1563. E-mail: balleine@psych.ucla.edu.

Copyright (C) 2000 Society for Neuroscience $0270-6474 / 00 / 208954-11 \$ 15.00 / 0$ be critically involved in encoding both the consequences of an action and changes in the incentive value of those consequences in hungry rats.

From this perspective, recent suggestions that the cortical targets of brainstem visceral and taste centers form a distributed memory system encoding biologically potent sensory events are of considerable interest (Braun, 1989; Rolls, 1994). Within this system, the ability of animals to encode the specific taste features of biologically potent events appears to depend on a region of the insular cortex centered on the median artery above the rhinal sulcus, referred to as the gustatory cortex (GC) (Braun et al., 1982; Kosar et al., 1986a,b). The best evidence for this claim comes from the finding that damage to this region strongly attenuates the impact of a conditioned taste aversion treatment on the incentive value of foods and fluids (Braun et al., 1982; Braun, 1989). This effect on taste aversion learning is unlikely to be attributable to changes in the ability of animals to detect the taste features of foods and fluids themselves; taste thresholds for each of the primary tastes appear normal in GC-lesioned rats and do not differ from unoperated control groups (Braun et al., 1982), nor does the sensitivity of GC-lesioned rats to the effects of emetic treatments appear to be modified because they display a normal ability to develop potentiated aversions to odors (Kiefer et al., 1984). Therefore, Braun (1990) proposed that GC lesions induce a form of taste agnosia, hypothesizing that the GC provides animals with the ability to recall taste features over the delay between taste detection during consumption and detection of emesis (or other post-ingestive effects), thereby allowing the formation of an association between taste and illness.

Given the evidence that taste features of the instrumental outcome play a critical role in changes in incentive value induced by outcome devaluation, the "taste memory" hypothesis of GC function predicts that lesions of this structure should attenuate the impact of outcome devaluation by sensory-specific satiety on instrumental performance in hungry rats. Furthermore, this view specifies that the source of any deficit in sensitivity to outcome devaluation should lie in the inability of lesioned rats to recall the devalued taste and not in the detection of the primary taste itself or in the assignment of incentive value to the taste. Consequently, any effect of GC lesions on devaluation should be confined to a situa- 
tion in which the rat is forced to recall changes in outcome value to formulate a course of action. The aim of this series of experiments was to investigate this claim by characterizing the effect of cellbody lesions of the GC on instrumental conditioning.

\section{MATERIALS AND METHODS}

\section{Experiment 1}

\section{Subjects and apparatus}

Subjects were 20 male Hooded Lister rats (Harlan Olac, Bicester UK). They were housed in squads of four in a temperature- and humiditycontrolled room with lights on from 8:00 A.M. to 8:00 P.M. Upon delivery, rats were maintained with access to food and water ad libitum. They were then operated on, at which point they weighed at least $300 \mathrm{gm}$. After surgery, the animals were returned to their home cages for a period of $10 \mathrm{~d}$ recuperation. At this point and for all stages of behavioral testing, the animals were shifted to a $22.5 \mathrm{hr}$ food deprivation schedule, under which they received access to food for $1.5 \mathrm{hr}$ each day in their home cage at least $2 \mathrm{hr}$ after behavioral testing with water available ad libitum.

Instrumental training and testing was conducted in four Campden Instruments (Manchester, UK) operant chambers. Each chamber was equipped with a recessed magazine, a retractable lever, and a chain. The magazine was positioned in the center of the front wall and could be entered via a flap door, which was attached to a microswitch. The lever and chain (which was lowered through the ceiling from a microswitch) were positioned symmetrically to the right and left side of the magazine flap, respectively. The chambers were also fitted with a pellet dispenser and a peristaltic pump, both of which were programmed to deliver the instrumental outcomes into the recessed magazine. The outcomes used were a $45 \mathrm{mg}$ Noyes pellet (formula A) and $0.05 \mathrm{ml}$ of a $20 \%$ solution of maltodextrin (Cerestar Ltd., Manchester, UK). Each chamber was illuminated by a $3 \mathrm{~W}$ house light mounted in the center of the front panel above the magazine. A BBC microcomputer equipped with the SPIDER extension for on-line control (Paul Fray Ltd., Cambridge, UK) controlled the equipment and recorded lever presses and chain pulls. For the presentation of the outcomes outside the operant chambers, eight feeding cages were used. These were molded plastic boxes, $30 \times 13 \times 11 \mathrm{~cm}$ in size, with wire mesh ceilings. Pellets were given in small glass dishes placed inside these cages, whereas maltodextrin was given through calibrated drinking tubes inserted through a hole in the wire mesh ceiling.

\section{Surgical procedures}

Animals were anesthetized using a barbiturate-alcohol preparation $(0.3$ $\mathrm{ml} / 100 \mathrm{gm}$ ). After being marked for identification and shaved, they were placed in the stereotaxic frame (David Kopf Instruments, Tujunga, CA), and an incision was made into the scalp to expose the skull. The incisor bar was then adjusted to the level-head position. Rats in group GC $(n=10)$ received intracranial injections of $1.0 \mu \mathrm{l}$ of $0.09 \mathrm{M}$ quinolinic acid dissolved in PBS at the following coordinate sites: anteroposterior (AP), +1.2; mediolateral (ML), \pm 3.0 ; and dorsoventral, 5.0 with the inf usion needle lowered at an angle of $20^{\circ}$ in the ML plane. One injection was given on the left side and a second on the right side of the midline, using level-head coordinates derived from the stereotaxic atlas of Paxinos and Watson (1986). The injections were made using a $10 \mu \mathrm{l}$ Hamilton syringe through a 30 gauge injection cannula, which was glued into a 23 gauge sleeve for support. The toxin was infused at the rate of $0.33 \mu \mathrm{l} / \mathrm{min}$. The cannula was then left in place for $3 \mathrm{~min}$ to allow diffusion of the toxin away from the cannula tip before being raised.

To control for the effects of anesthesia, being placed in the stereotaxic instrument, skull holes, and the lowering of the injection cannula into the brain, the behavior of the lesioned animals was compared with that of sham-operated rats. For the rats in group sham $(n=10)$, exactly the same surgical procedure was conducted, but the injection cannula was filled with PBS alone and lowered to the same position as for the GC group, but no fluid was injected.

\section{Histological procedures}

At the end of the experiment, the animals were killed using a lethal barbiturate overdose and then perfused transcardially with $0.9 \%$ saline followed by $10 \%$ formalin solution. The brains were stored in $10 \%$ formalin solution for $48 \mathrm{hr}$ before being transferred to a $25 \%$ sucrose solution. Over a period of days, the brains were allowed to sink in the sucrose solution before $60 \mu \mathrm{m}$ frozen coronal sections were cut throughout the region of the GC, mounted on glass slides, and stained with cresyl violet. Slides were examined for extent of lesion by microscopically examining sections with reference to the stereotaxic atlas of Paxinos and Watson (1986). Histological assessment was conducted by comparing lesioned brains with the sham brain and by looking for the following features: gross morphological changes, such as holes and tissue collapse; the position and extent of gliosis and scarring; the cannula tract and injection placement; and signs of neuronal cell body shriveling and loss.

\section{Procedure}

Except where indicated, the rats were run twice daily in five squads of four, each squad containing subjects from both lesion conditions, counterbalanced for operant box.

Instrumental acquisition. Three days after the shift to a food deprivation schedule, the behavioral phase began with $3 \mathrm{~d}$ of magazine training in the operant chambers. Pellets and maltodextrin were delivered noncontingently into the magazine on an random time $30 \mathrm{sec}$ schedule in two separate sessions. Throughout the experiment, each session began with the onset of the house light and terminated with its offset after $20 \mathrm{~min}$. The assessment of instrumental acquisition began on day 4. Action-outcome assignment was counterbalanced such that, for five animals in group GC and five in group sham, pressing the lever delivered the pellets and pulling the chain delivered the maltodextrin; for the remaining animals in each group, the action-outcome assignments were reversed. Throughout training, the rats were given two separate training sessions each day: one on the lever alone and the other on the chain alone, with the action that was trained first on each day alternating from one day to the next. At no stage were the lever-pressing and chain-pulling actions explicitly shaped by the experimenter. During this phase, animals were trained on a fixed interval (FI) $20 \mathrm{sec}$ schedule, and this training continued on each action until each animal had earned 100 of each outcome, at which point the acquisition phase terminated. The effect of the delivery of each outcome on the number of actions performed before the delivery of the next outcome was used as a measure of the rate of acquisition.

During the acquisition phase, two animals in group sham and two in group GC failed to acquire lever pressing and/or chain pulling and were discarded from the experiment. In both groups, the animals were assigned to different action-outcome relationships, and so the groups, both now with $n=8$, remained balanced for this factor (four in each action-outcome assignment per group).

Instrumental training. On the day after the acquisition assessment was terminated, animals were trained to lever press and chain pull on a constant probability schedule that delivered the appropriate outcome with a fixed probability for the first response in each second again with each action trained separately in each session. This probability was 0.25 in the first session, 0.1 in the following two sessions, and 0.05 in the next eight sessions. Action-outcome assignment was the same as that in the acquisition phase. Again, at no stage were the lever-pressing and chain-pulling actions explicitly shaped by the experimenter. This constant probability schedule approximates to a random ratio (RR) schedule with a mean ratio parameter increasing from 4 through 10 , to 20 responses.

Outcome devaluation: extinction test. The devaluation treatment was conducted on the day after the final instrumental training session. This was accomplished by prefeeding the rats with one of the two outcomes for 60 min in the feeding cages. The allocation of outcomes to each rat for the prefeeding was counterbalanced within each lesion group, for both the action whose outcome was devalued (i.e., lever vs chain) and the outcome devalued (i.e., pellets vs maltodextrin). Thus, in both group lesion and group sham, for four rats, the lever outcome was devalued, whereas for four, the chain outcome was devalued; and for four rats, pellets were devalued, whereas for four rats, maltodextrin was devalued. Immediately after this treatment, the rats were placed in the operant chambers for the choice extinction test. In this test, both the lever and the chain were available, but neither of the two outcomes was delivered.

Outcome devaluation: reward test. The day after the extinction test, the animals were retrained on the two manipulanda in separate sessions on the RR 20 schedule. On the next day, the rats were given a reward test conducted with both the levers and chains present. This test differed procedurally from the devaluation treatment and extinction test only to the extent that the two outcomes were delivered as a consequence of instrumental performance. In this session, the two outcomes were delivered on independent ratio schedules, with each outcome earned on an RR 20 schedule (i.e., with a probability of 0.05 ). Before this second test, the rats consumed the same outcome that they had been given before the extinction test for $1 \mathrm{hr}$ in the feeding cages.

\section{Statistics}

In this experiment and in all subsequent studies, an $\alpha$ level of 0.05 was used to assess the statistical significance of the data analyses.

\section{Experiment 2}

\section{Subjects and apparatus}

The subjects and apparatus were the same as those used in experiment 1.

\section{Procedure}

After the reward test of experiment 1, all rats received two sessions of retraining on each of the two manipulanda in separate sessions with each outcome delivered with a probability of 0.05 for the first response in each second, as in the training phase of experiment 1 . On the following day, the contingency assessment began. The rats continued to be trained on the two manipulanda with the appropriate paired outcome delivered with a probability of 0.05 in separate $30 \mathrm{~min}$ sessions each day. They earned the same outcomes as in experiment 1, but, in addition, one of the two outcomes was also delivered unpaired in each of the sessions, such that one of the 
action-outcome contingencies was degraded and the other was not. Thus, for each subject, the unpaired outcome was the same as the paired outcome in one of the daily sessions and different from the paired outcome in the other. These unpaired outcomes were also delivered with a probability of 0.05 but in each second without a response. Within each group, the type of unpaired outcome (food pellets vs maltodextrin solution) delivered was counterbalanced with respect to the action-outcome assignment. Thus, for half of the animals trained to lever press for pellets and to chain pull for maltodextrin, the unpaired outcome was pellets, whereas for the other half, it was maltodextrin, and likewise for the animals that earned maltodextrin on the lever and pellets on the chain. The contingency assessment lasted for four sessions on each action conducted on successive days using this schedule. On the fifth day, responding on both manipulanda was extinguished in separate sessions in the absence of any outcomes.

Unless otherwise stated, the procedures used in experiment 2 were the same as those used in experiment 1 .

\section{Experiment 3}

\section{Subjects and apparatus}

Subjects were 20 experimentally naive male Hooded Lister rats (Harlan Olac). They were housed and maintained as described in experiment 1.

The apparatus used for instrumental training and testing was the same as that described for experiment 1. For the incentive learning phase (see below), the eight feeding cages described in experiment 1 were used. Again, pellets were given in small glass dishes placed inside these cages, whereas maltodextrin was given through calibrated drinking tubes inserted through a hole in the wire mesh ceiling.

\section{Surgical procedures}

The surgical procedures for both the sham and lesioned groups, including lesion coordinates and the concentration and volume of the quinolinic acid infused, were exactly as described in experiment 1.

\section{Histological procedures}

Histological procedures were exactly as described in experiment 1.

\section{Procedure}

The experiment was conducted in four stages: (1) instrumental training; (2) incentive learning; (3) extinction test; and (4) reacquisition tests. Except where indicated, the rats were run twice daily in five squads of four, each squad containing subjects from both lesion conditions, counterbalanced for operant box. Before the start of instrumental training, rats were placed on the $22.5 \mathrm{hr}$ food deprivation used in the previous studies.

Instrumental training. Magazine training was conducted as described for experiment 1 . On the $2 \mathrm{~d}$ after magazine training, animals were trained to chain pull and then to lever press with the appropriate reinforcer delivered on a continuous reinforcement schedule. Action-outcome assignments were counterbalanced such that, for five animals in each lesion group, lever pressing delivered food pellets and chain pulling delivered maltodextrin. The remaining animals in each group received the opposite actionoutcome assignment. After initial acquisition, animals were trained on the fixed probability schedules of reinforcement as described in experiment 1 . The probability of reinforcement was again 0.25 in the first session (i.e., RR 4), 0.1 in the following two sessions (i.e., RR 10), and 0.05 in the next eight sessions (i.e., RR 20). Again, at no stage were the lever-pressing and chain-pulling actions explicitly shaped by the experimenter. Each session began with the onset of the house light and terminated with its offset after 20 min.

During this phase, two animals in each lesion group (one from each action-outcome assignment in each group) failed to acquire one or other action and so were dropped from the experiment. Consequently, both the sham and lesioned groups were reduced to eight subjects per group.

Incentive learning. On the day after the final training session, the incentive learning phase began and continued for $6 \mathrm{~d}$. Animals received exposure to both outcomes with one reexposed in a high deprivation state (i.e., $22.5 \mathrm{hr}$ ) and the other reexposed in the low or undeprived state (i.e., $0 \mathrm{hr}$ ) with three sessions of reexposure given to each outcome. The reexposure treatment was counterbalanced within both lesion groups, both with respect to which outcome was reexposed in the undeprived state and for the order of deprivation conditions. To achieve this counterbalancing, for half of the animals assigned to each action-outcome condition in each group, the pellets were reexposed in the low deprivation state and the maltodextrin reexposed in the high deprivation state. The remaining animals in each group received the opposite deprivation state-outcome assignment. Furthermore, half of the animals in each deprivation state-outcome assignment condition were deprived in a low-high-low-high-low-high order of deprivation conditions, whereas the remaining animals were given the reverse order. Thus, for the first session of reexposure, half of the animals in each group were maintained on the $22.5 \mathrm{hr}$ food deprivation schedule after the final session of instrumental training. The remaining animals were given ad libitum access to their maintenance diet in their home cage from $\sim 2 \mathrm{hr}$ after the final training session until the first reexposure session the next day. After the first reexposure session, animals given this exposure when food deprived were now allowed ad libitum access to the maintenance diet in their home cage from $\sim 2 \mathrm{hr}$ after the reexposure session until the second reexposure session the next day. Animals given the first reexposure when undeprived were then deprived of food until the second reexposure session the next day. In this manner, six sessions of reexposure were given, three when food deprived and three when undeprived, with the deprivation conditions alternating in counterbalanced order.

The reexposure sessions themselves were each of 15 min duration. When animals were reexposed to the pellets, they were placed in the feeding cages, with the glass dishes each containing 50 of the food pellet outcome. When animals were reexposed to the maltodextrin solution, they were placed in the feeding cages for 15 min but the drinking tubes containing 50 $\mathrm{ml}$ of maltodextrin were attached to the cages for the final $5 \mathrm{~min}$ of this period only. After the final session of reexposure, all animals were given ad libitum access to their maintenance diet until the extinction test conducted the next day.

Outcome devaluation: extinction test. The choice extinction test was conducted on the day after the final reexposure session with all animals tested in the low deprivation state. This test was otherwise conducted exactly as that described for experiment 1 . Thus, both the lever and the chain were available, but no outcomes of any kind were scheduled during this session.

Reacquisition tests. Two hours after the extinction test, half of the animals in each group continued to receive ad libitum access to their maintenance diet in their home cage. The remaining animals were deprived of food until the first reacquisition session the next day. For this session, the performance of all of the animals was assessed on the chains and the levers in separate sessions on the RR 20 schedule using the same procedure as the instrumental training phase. Two hours after this session, rats that were food deprived for the first session were given ad libitum access to food, whereas rats that were undeprived were now food deprived until the second reacquisition test conducted the next day. In this manner, all animals received four reacquisition tests, two when undeprived and two when food deprived.

\section{RESULTS}

\section{Experiment 1}

Experiment 1 was divided into four stages: (1) instrumental acquisition; (2) instrumental training; (3) a test of specific satietyinduced outcome devaluation conducted in extinction; and (4) a test of specific satiety-induced outcome devaluation conducted with reward. After recovery from surgery, we maintained both group GC and their sham operated counterparts on a food deprivation schedule and trained them to perform two instrumental actions, lever pressing and chain pulling, in different sessions with one action earning access to food pellets and the other to a maltodextrin solution. To assess the effect of the lesion on specific satietyinduced outcome devaluation, we gave the rats $1 \mathrm{hr}$ exposure to one of the outcomes, immediately after which they were returned to the operant chambers and their tendency to lever press and chain pull was assessed in a choice extinction test. To assess whether any effect of the lesion observed in the extinction test was attributable to a deficit in memory rather than in devaluation per se, after a period of retraining, we conducted a second, rewarded choice test immediately after prefeeding on one of the outcomes. In this test, the action that delivered the outcome on which the rats were sated and the action that delivered the outcome on which they were not sated were the same as in training.

On the basis of our previous findings, we anticipated that sham animals would perform fewer of the action that, in training, delivered the devalued outcome than of the other action in both the extinction and the rewarded choice tests. If the GC is involved in encoding changes in incentive value of the instrumental outcome induced by the specific satiety treatment, lesions of the GC should be anticipated to reduce this outcome devaluation effect but only in the test conducted in extinction. Thus, in contrast to the extinction test, a reliable devaluation effect should be anticipated in the rewarded choice test when the two outcomes are actually presented and, hence, when the relative incentive value of each outcome need not be recalled but is available for direct assessment.

\section{Histology}

Figure 1 illustrates the largest and the smallest areas of lesion damage observed in experiment 1 , and Figure 2 provides a photomicrograph of a representative section taken from a lesioned rat. Generally, the lesion was ventral to somatosensory cortex and was found to be most extensive in the granular and dorsal agranular 
areas of the insular cortex. Damage to these areas was observed throughout the rostrocaudal extent of the GC region as defined previously (cf. Braun et al., 1982; Kosar et al., 1986a), with evidence of extensive bilateral cell loss and gliosis centered dorsal to the rhinal sulcus and ventrolateral to the external capsule. Damage to the ventral agranular area was also observed but was primarily confined to the region directly ventral to the site of infusion. Gross morphological changes became more evident as the lesion extended laterally, with holes and some tissue collapse noted in superficial cortical layers. Lesion damage was very similar in six rats in group GC but was found to be mildly asymmetrical in the two remaining rats, being less extensive and more medial (bordering the claustrum) on one side relative to the other. Although this suggested that the surface layers of the cortex may well have been spared in one hemisphere of these animals, there was, nevertheless, considerable overlap with the contralateral lesion as well as with the damage observed in the other rats. For this reason and because we had no a priori grounds for regarding this variability as critical, we did not feel justified in dropping these animals from the study. Because we could observe no evidence of damage to the insular cortex in any of the rats in group sham, the behavioral data from all of the rats in group GC and group sham that completed instrumental training were analyzed (i.e., $n=8$ per group).

\section{Instrumental acquisition}

The results of the instrumental acquisition phase are presented for group lesion and group sham in Figure 3 separately for the acquisition of lever pressing (left panel) and the acquisition of chain pulling (right panel). In general, it is clear that the FI $20 \mathrm{sec}$ schedule was successful in establishing slow and orderly acquisition of the two instrumental actions as assessed by the number of actions performed after the delivery of each outcome. Both actions were acquired at a similar rate, and the lesion did not have any consistent impact on either the rate of acquisition or asymptotic level of performance of the two instrumental actions, a conclusion that was supported by the statistical analysis.

For this analysis, a three-way mixed ANOVA was conducted with a between-subjects factor of group and within-subjects factors of action and of block, averaging the performance of each action into blocks of five outcomes. This analysis revealed no main effect of group $(F<1)$ or of action $\left(F_{(1,14)}=1.79\right)$, nor was the group $\times$ action interaction reliable $(F<1)$. A significant effect of block was found $\left(F_{(19,266)}=19.85\right)$, but neither the group $\times$ block $(F<1)$, action $\times$ block $\left(F_{(14,266)}=1.42\right)$, nor the three-way interaction $(F<$ 1) was reliable.

\section{Outcome devaluation: extinction test}

The results of the extinction test are presented in Figure 4 separately for the action that, in training, delivered the outcome subsequently devalued by specific satiety (i.e., the devalued action) and for the action trained with the outcome that remained valued (i.e., the valued action). The performance on the valued and devalued actions is presented separately for group sham (middle panel) and group GC (right panel). The data from the final training session on the RR 20 schedule are presented in the left panel (see below for discussion). The results from the extinction test for group sham are clear; the performance of the devalued action was markedly reduced compared with that of the valued action. Although performance generally declined over the course of the extinction session, from the very first 2 min period, a striking difference in performance was evident. Importantly, Group lesion did not show this difference. Indeed, in this group, no clear or consistent evidence of a devaluation effect emerged at any point during the extinction test. Again, performance declined over the course of extinction, but both actions appeared to be performed at a high but similar rate, with neither action revealing the effects of the specific satiety devaluation treatment.

For the statistical analysis, a three-way mixed ANOVA was conducted with a between-subjects factor of group and withinsubjects factors of devaluation, separating performance on the
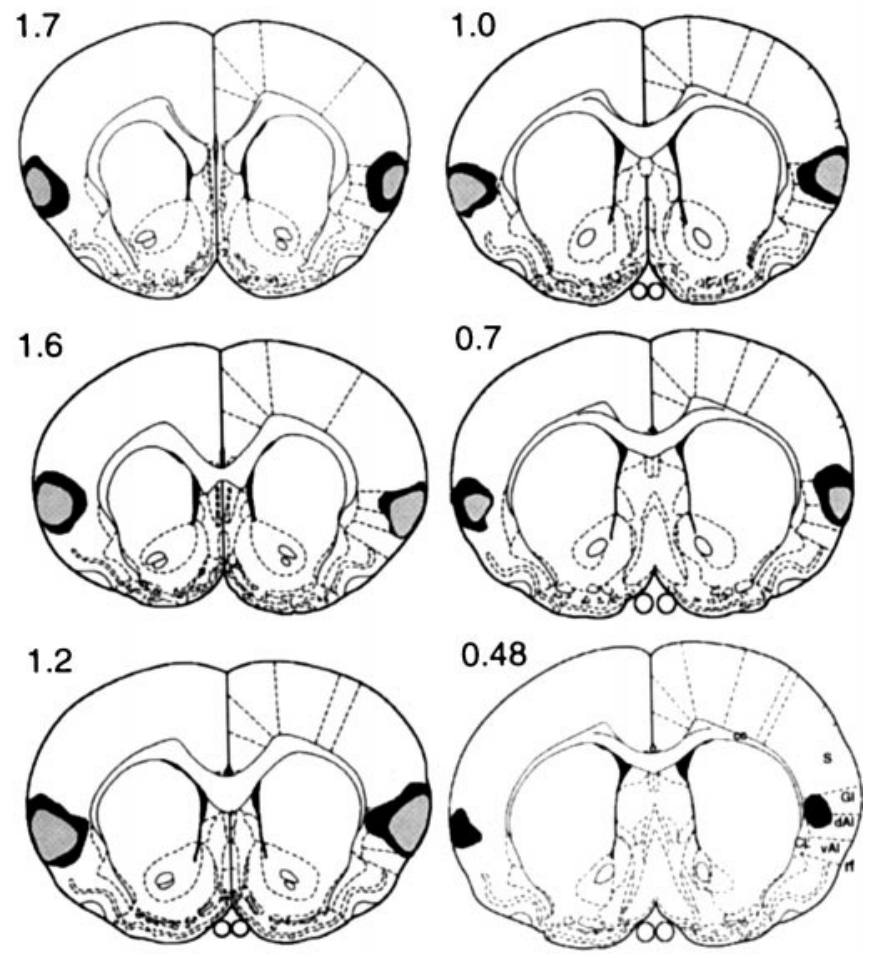

Figure 1. Experiment 1. Diagrams of coronal sections (0.48-1.7 mm anterior to bregma) on which the extent of cell loss observed after bilateral infusions of quinolinic acid aimed at the gustatory region of the insular cortex has been reconstructed from histology to reveal the largest (darker) and smallest (lighter) regions of damage induced in group GC. The lines drawn on the right-hand hemisphere extending laterally from the corpus callosum $(c c)$ and the claustrum $(C L)$ reflect divisions between (from the top) primary somatosensory $(S)$, granular $(G I)$, dorsal agranular $(d A I)$, and ventral agranular $(v A I)$ regions of insular cortex, respectively, as labeled on the lowermost section. $r f$, Rhinal fissure.

devalued action from that on the valued action, and of period, separating performance into 2 min periods. This analysis revealed no main effect of group $(F<1)$ but a main effect of devaluation $\left(F_{(1,14)}=8.96\right)$ and, most importantly, a significant group $\times$ devaluation interaction $\left(F_{(1,14)}=12.39\right)$. Simple main effects analysis conducted on this significant interaction revealed that, whereas performance on the valued action did not differ between groups $\left(F_{(1,14)}=2.69\right)$, performance of the devalued action with the animals in group GC performing at a significantly higher rate than those in group sham $\left(F_{(1,14)}=7.26\right)$. In addition, whereas a significant devaluation effect emerged in group sham $\left(F_{(1,14)}=21.2\right)$, no such effect was found in group lesion $(F<1)$. Finally, the overall analysis revealed effects of both period $\left(F_{(9,126)}=9.04\right)$ and a devaluation $\times$ period interaction $\left(F_{(9,126)}=5.13\right)$, confirming that performance generally declined over the extinction session and at a faster rate for the valued than for the devalued action.

These effects of a lesion of the GC on the outcome devaluation effect occurred only during the test and were not present in the training data. The data from the final training session on the RR 20 schedule are presented in the left panel of Figure 4. As is clear from that figure, performance between the two groups was very similar, as was their performance on the devalued and valued actions. Analysis of these data revealed no main effect of group or devaluation or any interaction between these factors $(F<1)$.

\section{Outcome devaluation: reward test}

The results of the reward test are presented in Figure 5, again separately for devalued and valued actions and for group sham (left panel) and for group GC (right panel). As in the extinction test, devaluation of the instrumental outcome induced a strong reduction in the performance of the devalued action in group sham. In 


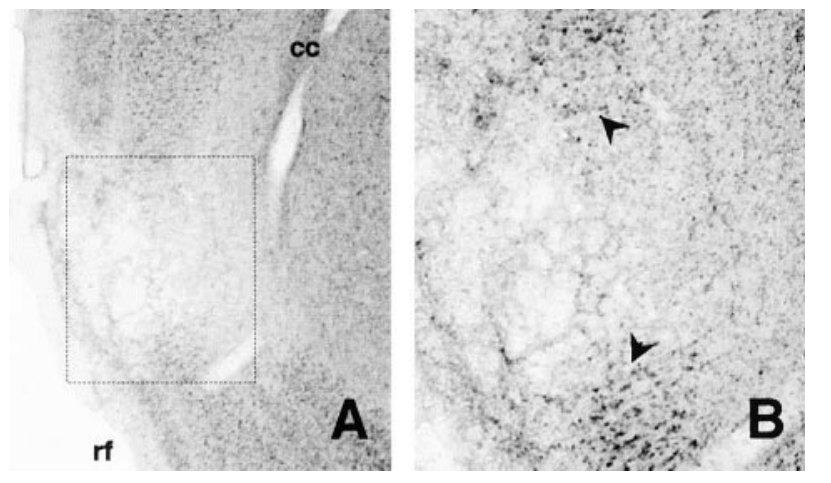

Figure 2. Experiment 1. Photomicrograph showing a Nissl-stained coronal section through the insular cortex ( $\sim 1.0 \mathrm{~mm}$ anterior bregma). $A$ shows this section in low magnification, whereas in $B$, the area enclosed by the dashed rectangle in $A$ has been enlarged to give a clearer indication of the lesion boundaries (arrows). cc, Corpus callosum; $r f$, rhinal fissure.

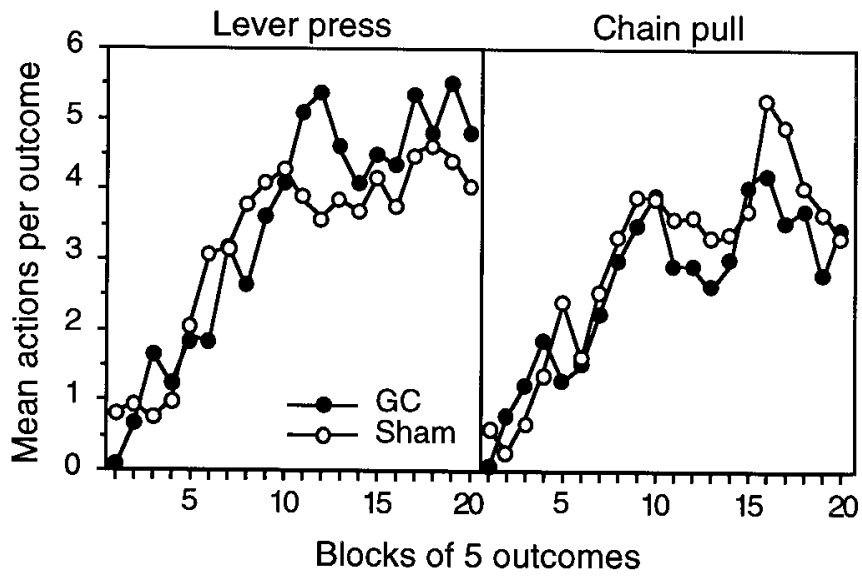

Figure 3. Experiment 1. The mean number of lever presses (left) and chain pulls (right) performed per outcome during instrumental acquisition on the FI 20 reinforcement schedule used in experiment 1. Data are averaged across blocks of five outcomes and are presented separately for group GC ( filled circles) and group sham (open circles).

contrast to the test conducted in extinction, however, this effect was also observed in group GC. A three-way mixed ANOVA found a significant effect of devaluation $\left(F_{(1,14)}=28.9\right)$ but no effect of group $\left(F_{(1,14)}=1.77\right)$ or a group $\times$ devaluation interaction $(F<1)$. Furthermore, there was no effect of period nor were any of the other interactions involving group, devaluation, or period significant $\left(\right.$ largest $\left.F_{(9,126)}=1.32\right)$.

Again, this effect of outcome devaluation was found on test and was not present in the retraining session conducted between the extinction test and the reward test. Comparable analysis of that training session revealed no effect of lesion, of devaluation, or any interaction between these factors $(F<1)$. Rate of performance on the devalued and valued actions, respectively, for the two groups was as follows: group sham, 23.4 and 22.1 actions per minute; group GC, 20.1 and 21.3 actions per minute.

The results of experiment 1 accord with the taste memory hypothesis of GC function advanced in the introductory remarks. Although lesions of the GC had no detectable effect on the acquisition or subsequent performance of either lever pressing or chain pulling for either outcome, they had a clear effect on the sensitivity of instrumental performance to the effects of outcome devaluation induced by a specific satiety treatment. Thus, in contrast to the differential performance observed in the sham-lesioned controls, GC-lesioned rats performed both actions at a similar rate and appeared unable to integrate the current incentive value of the instrumental outcomes, induced by the prefeeding, with the specific action-outcome relationships to which they were exposed during training.

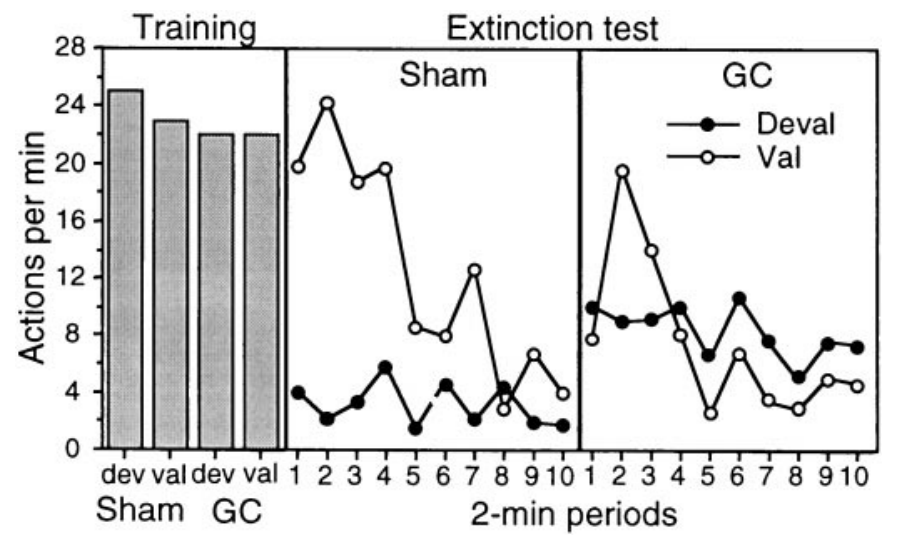

Figure 4. Experiment 1. The number of lever presses and chain pulls (i.e., actions) per minute during instrumental training (left) and during the choice extinction test conducted after one of the training outcomes was devalued by a specific satiety treatment. Data from the extinction test are presented for group sham (middle) and group GC (right) averaged across 2 min periods with performance of the action that previously delivered the pre-fed, i.e., devalued (Deval), outcome ( filled circles) presented separately from performance of the action that had delivered the non-pre-fed, i.e. valued ( $\mathrm{Val})$, outcome (open circles) for each group.

This effect of the lesion was, however, only observed in extinction and was not found in the reward test; when the devalued outcome was delivered contingent on performance in the reward test, the lesioned rats demonstrated a clear preference for the action delivering the nondevalued outcome relative to the other action. This finding is important because it helps to rule out several alternative accounts of the deficit observed in the extinction test. Thus, differential performance of the lesioned rats in the reward test suggests that the deficit in extinction was not attributable to insensitivity to the specific satiety treatment nor was it attributable to an inability to discriminate either the two instrumental outcomes or the two instrumental actions. Consequently, the results of experiment 1 suggest that the lesioned rats failed to encode the taste features of the two instrumental outcomes with the incentive value of these outcomes and, therefore, after sensory-specific satiety, they were unable to recall the value of one outcome relative to the other and so formulate a course of action when forced to rely on their memory of relative outcome value in the extinction test. When the valued and devalued outcomes were actually presented in the reward test, however, there was no longer any need to rely on memory of the relative value of the two outcomes and, therefore, the lesioned rats were able to choose appropriately.

\section{Experiment 2}

There is, however, an alternative account of the results of experiment 1 that remains to be assessed before we can accept that the insular cortex plays a role in taste-mediated memory of incentive value. The devaluation effect observed for group sham in the first study demonstrates that performance of the instrumental actions in our procedure is, at least in part, based on knowledge of the action-outcome contingency. If the lesioned animals in this experiment were unable to encode this contingency, then they may have acquired instrumental performance using only an S-R mechanism. Therefore, performance during the extinction test would have been resistant to the outcome devaluation treatment. This account also explains the divergent results found in the extinction and reward tests if it is assumed that a reduction in the reinforcing efficacy of one or other outcome through prefeeding failed to maintain the $\mathrm{S}-\mathrm{R}$ association for the response trained with the pre-fed outcome in the reward test. Although such an account implicates the GC in contingency learning, its logical possibility makes it necessary to assess whether the GC-lesioned animals are able to encode specific action-outcome relationships.

In fact, a number of investigators have established that, in intact animals, instrumental performance is sensitive to the contingency 


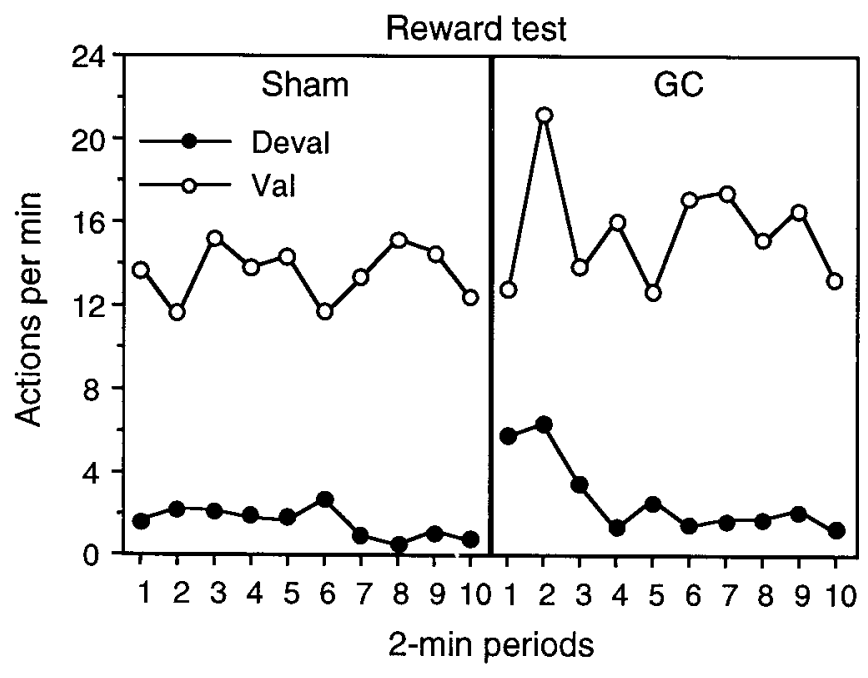

Figure 5. Experiment 1. The number of lever presses and chain pulls (i.e., actions) per minute during the choice reward test conducted after one of the training outcomes was devalued by a specific satiety treatment. In contrast to the extinction test, performance of lever-press and chain-pull actions delivered the training outcomes on independent random ratio schedules. Data from the reward test are presented for group sham (left) and group GC (right) averaged across 2 min periods with performance of the action that previously delivered the pre-fed, i.e., devalued (Deval), outcome ( filled circles) presented separately from performance of the action that had delivered the non-pre-fed, i.e., valued ( Val), outcome (open circles) for each group.

or causal relationship between performance of an action and delivery of its specific outcome (Hammond, 1980; Colwill and Rescorla, 1986; Dickinson and Mulatero, 1989; Corbit and Balleine, 2000). For example, Corbit and Balleine (2000) found that, when the action-outcome contingency was degraded for one of two action-outcome relationships by delivering one outcome with an equal probability both after performance of the appropriate action and in periods when the action was not performed, animals selectively decreased their performance of the action for which the contingency had been degraded but continued to perform the other action. In experiment 2, this same procedure was used to assess whether the rats in group GC in experiment 1 were able to encode the action-outcome contingencies.

To assess contingency sensitivity, after retraining with the same action-outcome assignment used in experiment 1 , the rats were shifted to a schedule in which the probability of outcome delivery after performance of each action remained the same, but, in addition, one of the two outcomes was also delivered noncontingently with the same probability in each second without a response. As a consequence, one action-outcome contingency was degraded, whereas the other remained intact. Four sessions of contingency assessment were conducted in this manner, after which extinction tests were conducted on both the lever and the chain to assess any contribution to performance made by increased satiety on one outcome relative to the other.

We anticipated, based on previous reports, that the animals in group sham would demonstrate sensitivity to degradation of one instrumental action-outcome contingency and reduce their performance of an action when its outcome is the same as the outcome delivered noncontingently. Likewise, if GC-lesioned animals are able to encode the specific action-outcome contingencies, then we should anticipate a similar effect to that predicted in group sham. If, however, the results of experiment 1 reflect the fact that group GC were unable to encode specific action-outcome contingencies, then no selective effect of this treatment should be anticipated and so no difference should emerge in the performance of the two actions.

The results from the contingency assessment are presented in Figure 6, separately for each of the four sessions of this phase (left four panels) and for the extinction test (far right panel). The data for group sham (top panel) and group lesion (bottom panel) are also presented separately for the actions for which the paired and unpaired outcomes were either the same (Same) or different (Diff). Over the four sessions of training, animals reduced their performance of the same action more than the different action, suggesting that the action-outcome contingency was successfully degraded by this manipulation. This result was further confirmed in the extinction test in which this pattern of responding clearly persisted when no outcomes were presented. Finally, and most importantly, the effect of degrading the instrumental contingency was similar in both group sham and group GC, suggesting that the lesioned animals were capable of encoding the specific action-outcome contingencies.

This description of the data were confirmed by the statistical analysis. A four-way mixed ANOVA was conducted on the data from the four sessions of contingency assessment with a betweensubjects factor of group and within-subjects factors of contingency, separating performance of the same and different actions, session, and 3 min periods in each session. This analysis revealed a main effect of contingency $\left(F_{(1,14)}=11.90\right)$ but neither a main effect of group nor a group $\times$ contingency interaction $(F<1)$. Furthermore, simple main effects analyses revealed a significant effect of contingency in both group sham $\left(F_{(1,14)}=6.61\right)$ and in group lesion $\left(F_{(1,14)}=5.34\right)$. In addition, there was a main effect of session $\left(F_{(3,42)}=35.29\right)$ and an interaction between session and contingency $\left(F_{(3,42)}=2.99\right)$, indicating that the effect of contingency developed over sessions. There was also an effect of period $\left(F_{(9,126)}=8.22\right)$ and a session $\times$ period interaction $\left(F_{(9,126)}=3.66\right)$, demonstrating that overall performance declined within a session, with this effect being more evident in earlier than in later sessions. None of the other higher order interactions were significant (maximum $F=1.25$ ).

A two-way analysis of the data from the extinction test was conducted using factors of group and of contingency. This analysis revealed a main effect of contingency $\left(F_{(1,14)}=27.41\right)$ but not an effect of group $\left(F_{(1,14)}=1.44\right)$ or a significant group $\times$ contingency interaction $(F<1)$. Simple main effects analysis again revealed a significant effect of contingency in both group sham $\left(F_{(1,14)}=\right.$ $14.10)$ and in group lesion $\left(F_{(1,14)}=13.34\right)$.

Overall, these data are consistent with the suggestion that GClesioned animals are able to encode the action-outcome contingencies to which they are exposed and in a manner that does not differ from sham animals. No evidence was found in experiment 2 to support the contention that the deficit in outcome devaluation observed in experiment 1 in GC-lesioned rats was caused by an inability to encode the action-outcome contingency. The pattern of results found in experiments 1 and 2 is consistent, therefore, with the conclusion that GC-lesioned animals are able to detect and discriminate different actions, different outcomes, and different contingencies between actions and outcomes. Furthermore, whereas they are able to detect differences in the incentive value of taste features of instrumental outcomes when these are presented, it appears that they are unable to recall these relative incentive values and so modify performance of their actions in the absence of the outcomes themselves. As a consequence, these results accord with the suggestion that the insular cortex forms a part of an incentive memory system that encodes the incentive value assigned to food outcomes in instrumental conditioning.

\section{Experiment 3}

The results of experiments 1 and 2 clearly indicate that the GC mediates the encoding of changes in the incentive value of the instrumental outcome but is not centrally involved in instrumental learning per se. In experiment 3 , we attempted to find additional evidence for this suggestion by exploring the effect of lesions of the GC on another manipulation of incentive value: that induced by a post-training shift in food deprivation.

Considerable recent evidence suggests that, rather than being directly sensitive to changes in motivational state, the rats' instrumental performance can be controlled primarily by the way motivational states affect the incentive value of the instrumental out- 


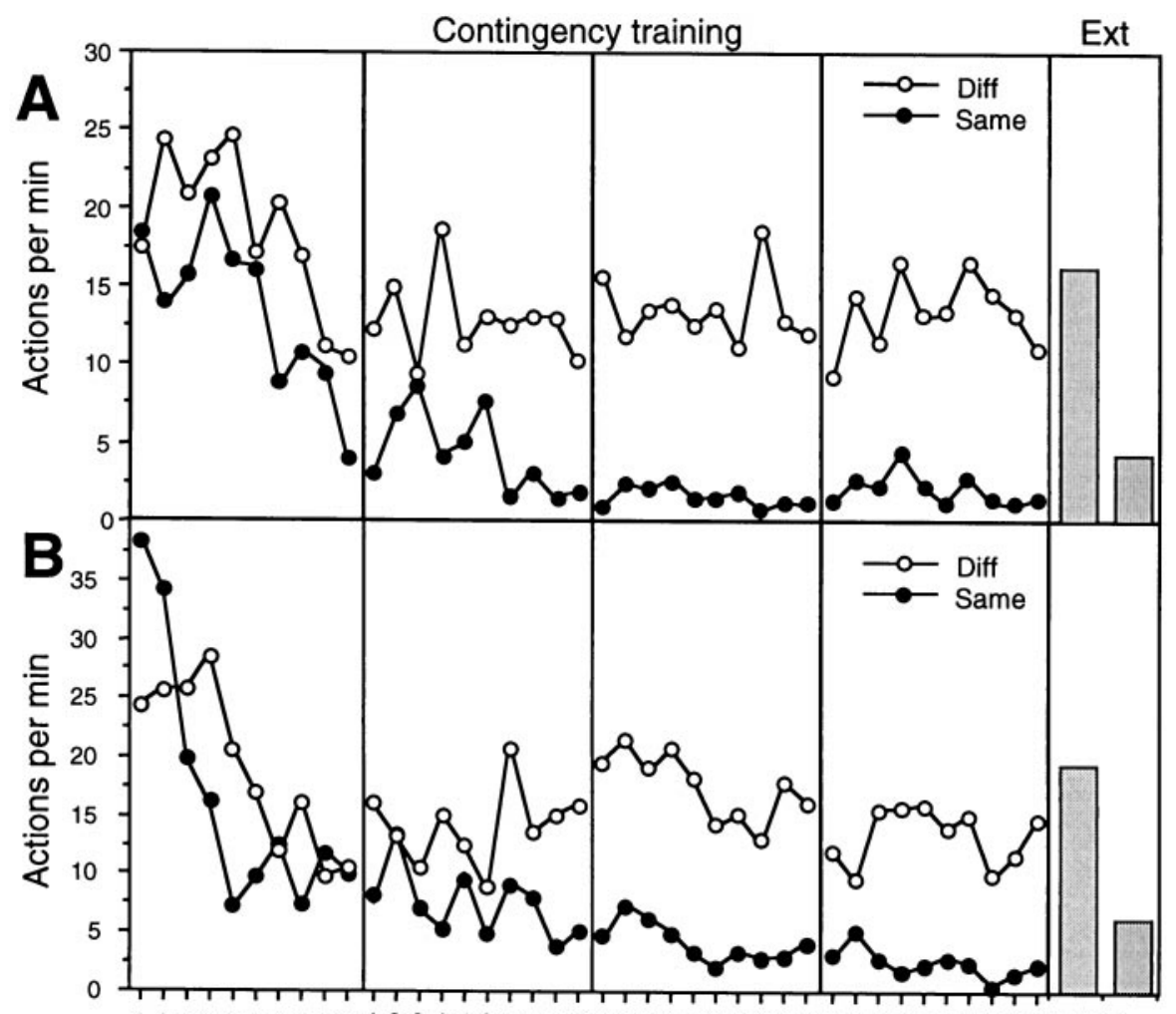

12345678910123456789101234567891012345678910 Diff Same

3-min periods/session
Figure 6. Experiment 2. Mean performance of lever-press and chain-pull actions per minute, averaged over 3 min periods, during each of the $4 \mathrm{~d}$ of contingency assessment (left four panels) and during the extinction test (right panel). Test performance is divided into two panels: $A$, showing the data from group sham; and $B$, showing the data from group GC. In this figure, performance of each action is presented separately in each panel according to whether the action-outcome contingency has been degraded, i.e., the outcome delivered by performing the action is the same as the one now delivered without performing the action (Same, filled circles) or has not been degraded, i.e., the outcome delivered by performing the action differs from that delivered without performing the action (Diff, open circles). In the panel illustrating the extinction test, the previously degraded action-outcome contingency remains designated as Same and the nondegraded as Diff, although no outcomes of any kind were presented in this test. come (for review, see Balleine, 2000). Thus, for example, in rats a reduction in food deprivation often only influences the performance of actions that gain access to a specific nutritive outcome if they have had the opportunity to consume the outcome after the shift, thereby allowing an evaluation of the effect of the new motivational state on the incentive properties of the outcome. We have referred to this as incentive learning (Balleine, 1992; Dickinson and Balleine, 1994).

In one study, for example, Balleine and Dickinson (1994) trained food-deprived rats to perform two actions, lever pressing and chain pulling, on a concurrent schedule with one action earning access to food pellets and the other to a maltodextrin solution. After training, the rats were given a number of reexposure sessions in which they were allowed to consume one of the instrumental outcomes in the training state, i.e., food deprived, and the other after a period of free feeding, i.e., in an undeprived state. The rats were then given a single, choice extinction test between the chain and the lever when undeprived with the test conducted in extinction. It was found that the animals performed significantly fewer of the action that, in training, had delivered the outcome to which they were subsequently reexposed in the undeprived state. Balleine and Dickinson (1994) interpreted this result as indicating that, during reexposure, the animals assigned a low incentive value to the outcome reexposed in the undeprived state, a differential evaluation that was then manifest in subsequent instrumental performance during the test. Consequently, this experiment suggests that, in instrumental conditioning, animals learn about the consequences of their actions and that motivational control of performance is then a matter of the way in which changes in deprivation act to modify the incentive value of those consequences.

From this incentive learning perspective, evidence that the GC is involved in encoding changes in the value of the taste features of the instrumental outcome suggests that this structure may encode changes in incentive value induced by a post-training change in primary motivation, such as that induced by a reduction in food deprivation. As a consequence, in experiment 3, we examined the effect of lesions of the GC on the sensitivity of the instrumental
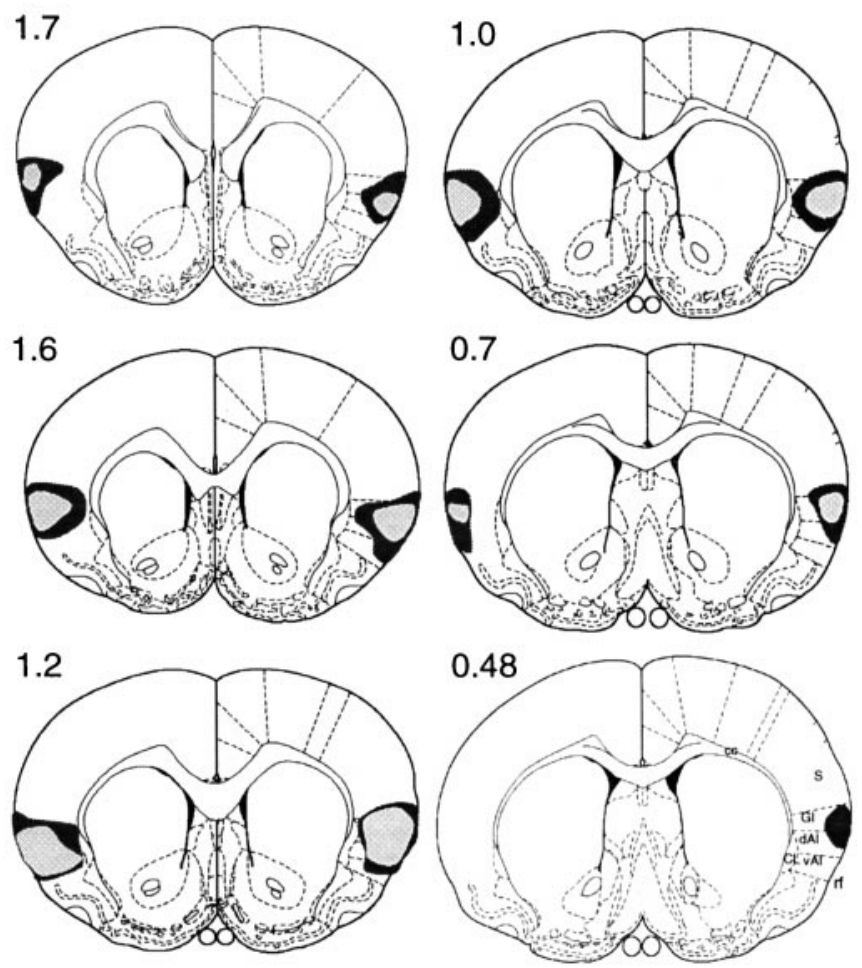

Figure 7. Experiment 3. Diagrams of coronal sections $(0.48-1.7 \mathrm{~mm}$ anterior to bregma) on which the extent of cell loss observed after bilateral infusions of quinolinic acid aimed at the gustatory region of the insular cortex has been reconstructed from histology to reveal the largest (darker) and smallest (lighter) regions of damage induced in group GC. Abbreviations are as for Figure 1. 


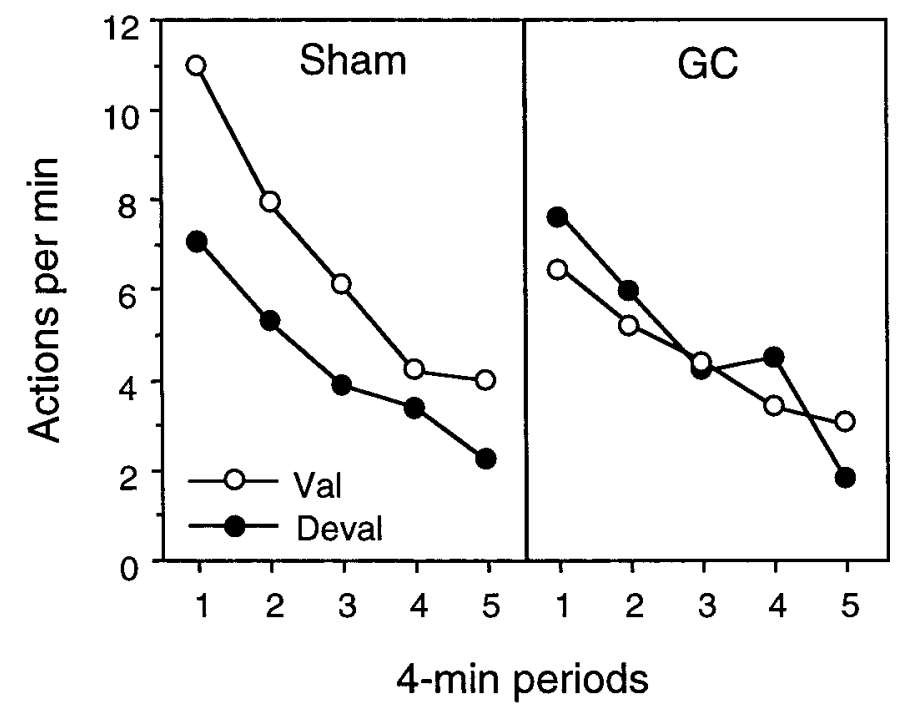

Figure 8. Experiment 3. Performance of lever-press and chain-pull actions averaged across 4 min periods in the choice extinction test conducted after a post-training reduction in food deprivation. Before this test, all of the rats were allowed to learn about the effect of the shift in food deprivation on the incentive value of one of the two food outcomes used in training by giving them brief consummatory contact with that outcome in the new, i.e., low deprivation, state. The effect of this treatment on choice performance in extinction is presented for group sham (left) and group GC (right) with the performance of the action that, in training, delivered the outcome reexposed in the low deprivation state (i.e., Deval, filled circles) plotted separately from performance of the other action (i.e., Val, open circles) in each group.

performance of rats to the effects of a post-training shift in primary motivation. Sham and lesioned rats were food deprived and trained to lever press and to chain pull, with one action delivering the food pellets and the other the maltodextrin solution. After this training, an incentive learning phase was conducted during which all rats were alternated between high $(22.5 \mathrm{hr})$ and low $(0 \mathrm{hr})$ levels of food deprivation for $6 \mathrm{~d}$. Half of the rats in each group were exposed to the food pellets when in the high deprivation state and maltodextrin when in the low deprivation state; the remaining animals received the opposite deprivation level: outcome assignment. After the incentive learning phase, rats were maintained in the low deprivation state and were then given a choice extinction test on the levers and chains.

In the choice extinction test, we anticipated that, in accord with previous studies (Balleine, 1992; Balleine and Dickinson, 1994), animals in the sham group would tend to assign a lower incentive value to the outcome to which they were reexposed when in the low deprivation state and so, when again in that state on test, would perform fewer of the actions that, in training, delivered this devalued outcome. If the GC mediates the encoding of changes in incentive value induced by shifts in motivational state, then, in the extinction test, rats with lesions of this area should be unable to recall the changes in incentive value induced by a shift to the low deprivation state and so should not show any differential performance of the two actions in the test.

This result would, however, be equally consistent with an impairment in sensitivity to the effects of a shift in food deprivation in the GC-lesioned animals. To assess this possibility, we conducted a final phase in which all of the rats were given four reacquisition sessions on the lever-press and chain-pull actions with performance delivering the outcomes assigned in training. Deprivation was alternated between the high and low state across these sessions.

\section{Histology}

The lesions in group GC were, generally, similar to those described in experiment 1 (Fig. 7). Again, extensive bilateral cell loss and gliosis was observed throughout the AP extent of the GC centered

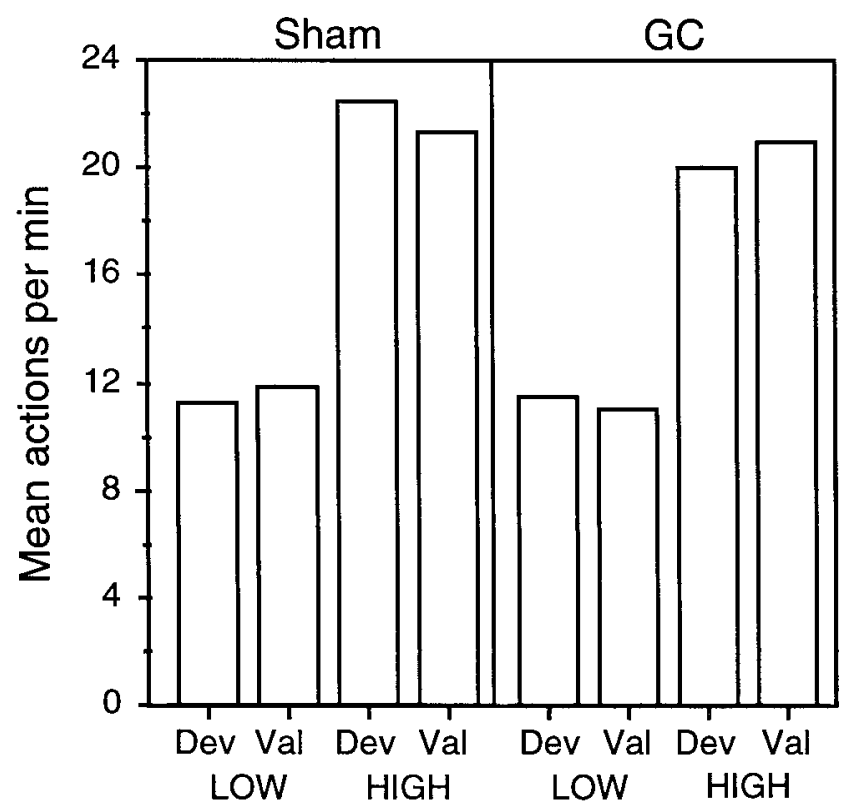

Figure 9. Experiment 3. Rate of performance of the lever-press and chain-pull actions in the reacquisition tests conducted after the extinction test. Rats were shifted between high and low levels of deprivation with the effect of these shifts assessed on the relative performance of the devalued $(\mathrm{Dev})$ and valued ( $\mathrm{Val})$ actions, as designated in the extinction test. The average rate of performance of each action was averaged across the two sessions conducted in each deprivation state and plotted separately for group sham (left) and group GC (right).

on the dorsal agranular area and extending into the granular area ventral to somatosensory cortex. Morphological changes were also observed to be similar to those described in experiment 1 . Once again, some asymmetry in the lateral extent of the lesion was noted in three animals but, again, there was considerable overlap with the location of the contralateral lesion in each of these rats as well as with the extent of the lesion observed in the other rats. Because similar variability did not appear to mitigate the effects of the lesion in experiment 1 , the data from all of the animals in group GC and group sham that completed instrumental training were analyzed (i.e., $n=8$ per group).

\section{Extinction test}

The results of the extinction test are presented in Figure 8 separately for the action that, in training, delivered the outcome subsequently reexposed in the low deprivation state (i.e., devalued) and for the other action trained with the outcome reexposed in the high deprivation state (i.e., valued). The performance on the valued and devalued actions is presented separately for group sham (left panel) and group GC (right panel). As indicated by Figure 8, in the extinction test, group sham performed the devalued action less frequently than the valued action, thereby replicating the incentive learning effect observed in previous studies (Balleine, 1992; Balleine and Dickinson, 1994). The same pattern did not emerge in group GC and, in this group, there was no difference in the performance of the two actions at any point during the extinction test.

This description was confirmed by the statistical analysis. A three-way mixed ANOVA was conducted with a between-subjects factor of group and within-subjects factors of devaluation, separating performance of the devalued action from that of the valued action, and of period, separating performance into 2 min periods. This analysis revealed no main effect of group $\left(F_{(1,14)}=1.02\right)$ or of devaluation $\left(F_{(1,14)}=1.39\right)$ but, most importantly, did reveal a significant group $\times$ devaluation interaction $\left(F_{(1,14)}=4.76\right)$. Simple main effects analysis conducted on this significant interaction revealed that, whereas performance on the valued action did not differ between groups $(F<1)$, performance did differ on the devalued 
action, with the animals in group GC performing this action at a significantly higher rate than those in group sham $\left(F_{(1,24)}=4.31\right)$. In addition, whereas a significant devaluation effect emerged in group sham $\left(F_{(1,14)}=5.64\right)$, no such effect was found in group GC $(F<1)$. Finally, the overall analysis revealed an effect of period $\left(F_{(9,126)}=3.86\right)$ but no other reliable effects.

These effects of lesions of the GC on outcome devaluation induced by a post-training shift in primary motivation occurred on test and were not present in the training data. Performance of the two groups was very similar, as was their performance on the devalued and valued actions during the final training session, and a comparable analysis of these data revealed no main effect of group or of devaluation or any interaction between these factors $(F<1)$. The mean number of actions per minute on the manipulandum that, in training, delivered the to-be-devalued and to-be-valued outcomes, respectively, were as follows: group sham, 23.7 and 22.6; and group GC, 21.3 and 22.4 .

\section{Reacquisition tests}

The reacquisition tests compared the effect of up and down shifts in food deprivation on rewarded instrumental performance in sham and lesioned rats. The results of these tests are presented in Figure 9 collapsed across sessions and session order. From this figure, it is clear that both group sham and group GC were sensitive to the shifts in food deprivation used in this experiment. Thus, the actions were performed at a higher rate when they were in a high deprivation state than when in a low deprivation state. More critically, this figure shows that the sensitivity of group GC to these shifts in deprivation did not differ from that displayed by group sham, indicating that the deficit in incentive learning was not produced by general insensitivity to the effects of shifts in food deprivation on the incentive value of foods. Finally, the performance of the actions designated "valued" and "devalued" in the extinction test (Fig. 9, Val, Dev) was not differentially affected by the shifts in deprivation in either group. Instead, it appears that the performance of both actions in group sham and group GC was strongly determined by deprivation state.

To assess this description of the data, a three-way ANOVA was conducted using a between-subjects factor of group and withinsubjects factors of deprivation state (high vs low) and devaluation (valued vs devalued action from the extinction test). This analysis revealed a significant main effect of deprivation state $\left(F_{(1,14)}=\right.$ 67.6) but no reliable main effect of group or devaluation (largest $\left.F_{(1,14)}=1.22\right)$. Furthermore, none of the interactions involving these factors approached significance (largest $F_{(1,14)}=2.1$ ).

As predicted by the taste memory account of GC function, lesions of the GC rendered instrumental performance relatively insensitive to the effects of outcome devaluation by a post-training reduction in food deprivation but only when assessed in an extinction test. GC-lesioned rats could clearly detect the changes in incentive value induced by these shifts in deprivation with performance in the reacquisition tests affected by deprivation state to a similar degree to that observed in the sham rats. Therefore, no evidence was found to suggest that the deficit in choice performance found in the extinction test was produced by a deficit in the processes that detect changes in incentive value induced by a shift in deprivation. Rather, the results of experiment 3 suggest that the lesioned rats fail to remember at the time of extinction testing what they learned during the incentive learning stage about the change in incentive value induced by shifts in motivational state. Specifically, what they fail to remember is the low incentive value assigned to the outcome reexposed under the low deprivational state, and consequently they continue to perform the action trained with this outcome at a level appropriate to a high incentive value.

The hypothesis that GC lesions cause a deficit in encoding changes in incentive value can be further refined based on evidence, described in the introductory remarks to this experiment, that food deprivation changes the incentive value of food outcomes by modifying the palatability of their taste features (cf. Balleine, 2000). This evidence suggests that the deficit in incentive learning induced by lesions of the GC may be specific to encoding changes in taste palatability. This view suggests that, whereas the GC is not critical for detecting either tastes or the effect of changes in deprivation on taste palatability, it is involved in encoding these events in memory during consummatory contact with the instrumental outcome. As a consequence, incentive learning is strongly attenuated in GC-lesioned rats because the lesion renders them unable to encode and so remember changes in the incentive value of foods induced by a shift in deprivation when forced to do so in the choice extinction test.

\section{DISCUSSION}

The aim of this series of experiments was to explore the role of the gustatory region of the insular cortex, the so-called gustatory cortex, in instrumental conditioning and, more specifically, instrumental outcome devaluation effects. No evidence was found in these experiments to suggest that this region plays a central role in the acquisition of instrumental conditioning or in the processes that detect and encode the instrumental action-outcome contingency. Nevertheless, these experiments do provide clear evidence that this region is involved in encoding changes in the incentive value of the instrumental outcome. More specifically, the results suggest that the gustatory cortex plays a critical role in "incentive memory," allowing animals to encode changes in incentive value based on changes in the palatability of taste features of the instrumental outcome detected during consummatory contact.

This conclusion is based on a number of findings. In experiment 1 , lesions of the GC had no effect on the rate of lever-press or chain-pull actions when these were acquired on an FI $20 \mathrm{sec}$ schedule of reinforcement, nor was evidence found that performance on the RR schedules was affected by the lesion as the response requirement was increased. Nevertheless, when one of the instrumental outcomes used during this training was devalued using a specific satiety treatment, a very potent effect of the GC lesion was found on instrumental performance in the subsequent choice extinction test. Sham animals could clearly recall which outcome had been devalued and could integrate that knowledge with the action-outcome relationships encoded during training because, on test, they performed fewer of the action that previously delivered the now-devalued outcome. In contrast, GC-lesioned rats showed no effect of devaluation and performed both actions at a similar rate and at a rate that was comparable with the valued action in group sham.

Importantly, this effect of the lesion only emerged when the test was conducted in extinction. When the effect of specific satietyinduced outcome devaluation was assessed in a reward test, i.e., a test conducted in the same manner as the extinction test except the outcomes were now delivered as consequence of instrumental performance, no effect of the lesion was detected. Thus, although GC-lesioned rats showed no devaluation effect in extinction, in the reward test they reduced performance of the action that delivered the devalued outcome relative to the other action in a manner that was indistinguishable from the sham group. This pattern of results is consistent with the view that GC lesions affect the rats' ability to recall the effects of devaluation treatments on outcome value without affecting their ability to detect these effects, a suggestion bolstered by the results of experiment 3 . In that experiment, we used a different procedure to devalue the instrumental outcome, i.e., devaluation by a post-training shift in primary motivation. Rats were trained while food deprived to lever press and chain pull for different food outcomes as in experiment 1 . After this training, the degree of food deprivation was reduced by providing access to their maintenance diet ad libitum, and the effect of this shift in deprivation on performance was then assessed in an extinction test. Before the test, however, and on the basis of our previous behavioral work, we first provided the rats with an opportunity for incentive learning by giving them several sessions in which they could consume small quantities of one instrumental outcome in the low deprivation state. This treatment has been reported selectively to devalue the outcome exposed in the undeprived state (Balleine 
and Dickinson, 1994; Balleine, 2000) and, in line with this suggestion, in extinction, rats in group sham performed fewer of the action that, in training, delivered the outcome exposed in the low deprivation state than of the other action. In contrast, the GClesioned rats did not show a selective devaluation effect and performed both actions at a similar rate. As in experiment 1 , this result was confined to extinction; the results of the reacquisition tests found that the GC-lesioned rats were as able as shams to detect the effect of shifts in food deprivation. Thus, in accord with experiment 1 , the results of experiment 3 suggest that lesions of the GC impair the ability of rats to recall changes in incentive value but do not appear to affect their ability to detect those changes.

Although consistent with the results of experiments 1 and 3 , it remains a possibility that, rather than mediating the memory for changes in outcome value, the GC is critical for encoding the instrumental action-outcome relationship during training. This account predicts that, after outcome devaluation, GC lesions will produce a deficit in choice performance, not because of a failure to encode or recall the reduced incentive value of an outcome but because of a failure to encode the causal relationship between an action and its specific consequences. We assessed this account in experiment 2 and found clear evidence against it. In this experiment, one action-outcome contingency was degraded by making the delivery of the outcome after the action and delivery of the outcome in the absence of the action equally probable. If the GC lesion renders rats unable to encode the specific action-outcome relationships to which they were exposed in training, the lesioned rats should have been relatively insensitive to noncontingent outcome delivery. Nevertheless, no evidence emerged to suggest that the lesioned rats differed from the shams in their ability to encode the instrumental action-outcome relationship. Both groups appeared to be similarly sensitive to degradation of the instrumental contingency.

\section{The role of the $\mathrm{GC}$ in incentive learning}

Together, the results of the current experiments suggest that, in instrumental conditioning, the GC is involved in recalling changes in incentive value induced by both specific satiety and a shift in primary motivation, changes that evidence suggests are mediated by incentive learning (Balleine, 1992; Balleine and Dickinson, 1998). Thus, it seems reasonable to propose that the GC is involved, generally, in the incentive learning process, i.e., a learning process through which changes in incentive value are encoded during direct consummatory experience. Nevertheless, this involvement is likely to be limited to situations in which changes in incentive value are based predominantly on changes in the evaluation of taste features of the instrumental outcome.

Balleine and Dickinson (1998) demonstrated that outcome devaluation by specific satiety can be mediated solely by the taste of a food outcome, which suggests that the incentive value in this situation is predominantly assigned based on representation of its taste features in this situation. If, therefore, an animal is unable to encode these taste features as an aspect of the representation of the instrumental outcome stored in memory, then the incentive value assigned to these features should also fail to be encoded in memory, and an animal required to establish the current incentive value of an outcome by interrogating its encoded outcome representation should have difficulty in doing so. This account of the results of the current experiments suggests that the failure to find an effect of outcome devaluation treatments in the GC-lesioned rats was not produced by either a reduction in sensitivity to devaluation or interference with instrumental learning processes. Rather, it is proposed that GC-lesioned animals are unable to encode the taste features of foods or fluids within their mnemonic representation of the instrumental outcome. The rats appear, therefore, to be insensitive to devaluation treatments that act by changing the palatability of taste features but only in situations that require an animal to retrieve the current value of an outcome from memory to formulate a course of action.
The hypothesis proposed based on this analysis is, therefore, that the gustatory region of the insular cortex operates to encode the taste features of the instrumental outcome as an aspect of the representation of that outcome in memory. It appears, therefore, that the GC mediates the encoding of one of the several sensory features (e.g., odor, texture, temperature, and visual properties) on which representation of the instrumental outcome and the assignment of incentive value could be based, albeit the most salient of these elements with respect to the assignment of incentive value to nutritive outcomes. As such, it seems reasonable to propose that the GC acts as part of a distributed memory system involving closely related cortical areas, such as somatosensory, visual, and olfactory regions, damage to all of which appear to generate specific sensory agnosias (Meyer, 1984; Braun, 1989) that together may provide the basis for the formation of a rich sensory representation of specific instrumental outcomes.

Finally, considerable evidence, too much to review in detail here (cf. Dickinson and Balleine, 1994; Balleine, 2000), has accumulated to support the view that incentive learning modifies the incentive value assigned to food and fluid outcomes by allowing animals to learn about a change in the affective response elicited by consummatory contact with those outcomes. The fact that, in the current studies, rats with lesions of the GC were capable of detecting changes in outcome value suggests that the $\mathrm{GC}$ is not critically involved in the presentation of affect. The evidence that GClesioned rats were unable to recall changes in value does, however, suggest that, after outcome devaluation, the GC functions to allow the changes in affective response elicited by foods and fluids to be encoded with the taste features of those events. Thus, although changes in incentive value based on changes in the palatability of taste features induced by devaluation treatments are not detected by the GC, it appears likely that it is involved in encoding those taste features in memory. As such, encoding of the incentive value of the outcome in memory must require, at the very least, the integration of taste memory, involving the GC, with other structures sensitive to the affective significance of nutritive outcomes. In this regard, it is worth noting that the GC maintains relatively rich connections with the amygdala (Sripanidkulchai et al., 1984; Yamamoto et al., 1984), a structure often implicated in emotional processing (Jones and Mishkin, 1972; Aggleton and Passingham, 1981). Indeed, because several recent studies have implicated both the agranular insular region of the prefrontal cortex and the amygdala in sensory specific satiety in primates (Malkova et al., 1997; Baxter et al., 2000), the role of this projection in instrumental conditioning generally and in incentive learning in particular would appear to offer a fruitful basis for future investigation.

\section{REFERENCES}

Aggleton JP, Passingham RE (1981) Syndrome produced by lesions of the amygdala in monkeys (Macaca mulatta). Physiol Psychol 95:961-977.

Balleine BW (1992) The role of incentive learning in instrumental performance following shifts in primary motivation. J Exp Psychol Anim Behav Process 18:236-250.

Balleine BW (2000) Incentive processes in instrumental conditioning. In: Handbook of contemporary learning theories (Mowrer R, Klein S, eds), pp 307-366. Hillsdale, NJ: Erlbaum

Balleine BW, Dickinson A (1994) Role of cholecystokinin in the motivational control of instrumental action in rats. Behav Neurosci 108:590-605.

Balleine BW, Dickinson A (1998) The role of incentive learning in instrumental outcome revaluation by sensory-specific satiety. Anim Learn Behav 26:46-59.

Baxter MG, Parker A, Lindner CC, Izquierdo AD, Murray EA (2000) Control of response selection by reinforcer value requires interaction of amygdala and orbito-prefrontal cortex. J Neurosci 20:4311-4319.

Braun JJ (1989) Experimental amnestic sensory agnosia: preoperative modulation. In: Preoperative events. Their effects on behavior following brain damage: comparative cognition and neuroscience (Schulkin J, ed), pp 233-253. Hillsdale, NJ: Erlbaum.

Braun JJ (1990) Gustatory cortex: definition and function. In: The cerebral cortex of the rat (Kolb B, Tees RC, eds), pp 407-430. Cambridge, MA: MIT.

Braun JJ, Lasiter PS, Kiefer SW (1982) The gustatory neocortex of the rat. Physiol Psychol 10:13-45.

Colwill RM, Rescorla RA (1985) Postconditioning devaluation of a rein- 
forcer affects instrumental responding. J Exp Psychol Anim Behav Process 11:120-132.

Colwill RM, Rescorla RA (1986) Associative structures in instrumental learning. In: The psychology of learning and motivation, Vol 20 (Bower GH, ed), pp 55-104. Orlando, FL: Academic.

Corbit LH, Balleine BW (2000) The role of the hippocampus in instrumental conditioning. J Neurosci 20:4233-4239.

Dickinson A, Balleine B (1994) Motivational control of goal-directed action. Anim Learn Behav 22:1-18.

Dickinson A, Mulatero CW (1989) Reinforcer specificity of the suppression of instrumental performance on a non-contingent schedule. Behav Process 19:167-180.

Donahoe JW, Burgos JE, Palmer DC (1993) A selectionist approach to reinforcement. J Exp Anal Behav 60:17-40.

Hammond LJ (1980) The effects of contingencies upon appetitive conditioning of free-operant behavior. J Exp Anal Behav 34:297-304.

Jones B, Mishkin M (1972) Limbic lesions and the problem of stimulusreinforcement associations. Exp Neurol 36:362-377.

Kiefer SW, Leach LR, Braun JJ (1984) Taste agnosia following gustatory neocortex ablation: dissociation from odor and generality across taste qualities. Behav Neurosci 98:590-608.

Kosar E, Grill HJ, Norgren R (1986a) Gustatory cortex in the rat. I. Physiological properties and cytoarchitecture. Brain Res 379:329-341.
Kosar E, Grill HJ, Norgren R (1986b) Gustatory cortex in the rat. II. Thalamocortical projections. Brain Res 379:342-352.

Malkova L, Gaffasn D, Murray E (1997) Excitotoxic lesions of the amygdala fail to produce impairment in visual learning for auditory secondary reinforcement but interfere with reinforcer devaluation effects in rhesus monkeys. J Neurosci 17:6011-6020.

Meyer DR (1984) The cerebral cortex: its role in memory storage and remembering. Physiol Psychol 12:81-88.

Paxinos G, Watson C (1986) The rat brain in stereotaxic coordinates, Ed 2. Sydney: Academic.

Robbins TW, Everitt BJ (1996) Neurobehavioural mechanisms of reward and motivation. Curr Opin Neurobiol 6:228-236.

Rolls ET (1994) Neural processing related to feeding in primates. In: Appetite: neural and behavioural bases (Legg CR, Booth DA, eds), pp 11-53. Oxford: Oxford UP.

Sripanidkulchai K, Sripanidkulchai B, Wyss JM (1984) The cortical projection of the basolateral amygdaloid nucleus in the rat: a retrograde fluorescent dye study. J Comp Neurol 229:419-431.

Thorndike EL (1911) Animal intelligence: experimental studies. New York: Macmillan.

Yamamoto T, Azuma S, Kawamura Y (1984) Functional relations between the cortical gustatory area and the amygdala: electrophysiological and behavioral studies in rats. Exp Brain Res 56:23-31. 